\title{
Depth-Dependence and Time-Dependence in Mechanical Behaviors of Articular Cartilage in Unconfined Compression Test under Constant Total Deformation"
}

\author{
Natsuko HOSODA $^{* *}$, Nobuo SAKAI ${ }^{* * *}$, Yoshinori SAWAE ${ }^{* * *}$ \\ and Teruo MURAKAMI ${ }^{* * *}$ \\ ${ }^{*}$ Graduate School of Systems Life Sciences, Kyushu University, \\ 744 Motooka, Nishi-ku, Fukuoka-shi, Fukuoka 819-0395, Japan \\ E-mail: natsuko@tribo1.mech.kyushu-u.ac.jp \\ ***Faculty of Engineering, Kyushu University, \\ 744 Motooka, Nishi-ku, Fukuoka-shi, Fukuoka 819-0395,Japan
}

\begin{abstract}
Articular cartilage has high water content from 70 to $80 \%$ and biphasic property. The structures of the tissue are inhomogeneous and anisotropy. Consequently, cartilage tissue shows complicated viscoelastic behavior to mechanical stimuli because of depth-dependent and time-dependent interstitial fluid flux and stress-strain behaviors. Therefore it is necessary to consider not only the average tissue property but also the local one to explain mechanical and functional behaviors. The aim of this study is to consider the effect of elastic modulus distribution on the mechanical behavior of cartilage tissue using experiments with visualization and two-dimensional finite element method (FEM).

In this study, we performed the compression test of the articular cartilage under the unconfined condition. First, on the basis of the distribution of Young's modulus in depth direction calculated from local strain at equilibrium in experiment, the stress and strain behaviors in articular cartilage were analyzed by biphasic theory.

Immediately after loading, the FEM analysis for deformed profiles of the model with depth-dependent Young's modulus corresponded to actual profiles, while the model with average value for Young's modulus showed inadequate deformed profiles. However, the total load-carrying capacity estimated in FEM for the former model is about one order lower than the experimental one measured by a load cell. Therefore, we provided time-dependence to elastic property to understand the complicated viscoelastic behavior during stress relaxation. Thus, the deformed shape profiles of the model immediately after loading and the total load-carrying capacity could satisfactorily correspond to measured data by considering depth-dependent and time-dependent variation of elastic property.
\end{abstract}

Key words: Articular Cartilage, Compressive Deformation, Finite Element Method, Biphasic Analysis, Depth-Dependence, Time-Dependence

\section{Introduction}

The human synovial joint possesses superior load-bearing and jointing functions with very low friction and low wear. Articular cartilage tissue plays an important role to maintain this function through whole life. The arthroidal joint forms a connection between two or more bones, and functions by supporting and transmitting loads. Cartilage has high water 
content from 70 to $80 \%$ and shows biphasic behaviors in which both solid and fluid properties should be considered.

One of the representative diseases on the synovial joints particularly for elder persons is osteoarthritis. Osteoarthritis causes degeneration and destruction of articular cartilage, which lead to the disturbance of motility. Articular cartilage degenerated by osteoarthritis decreases load-buffering capacity and leads to the bone deformation, the formation of osteophytes, and the subchondral bone induration and thickening. Especially in joints of lower extremities, there are many reports linking age and obesity to the osteoarthritis, or external injury and career to the occurrence of osteoarthritis. Consequently, mechanical factors are believed to play a crucial role in the pathogenesis of osteoarthritis.

As a new treatment method, tissue engineering has attracted attention in recent years. At the present stage, however, the mechanical function of tissue-engineered cartilage has not reached the level of native cartilage. It is noted that mechanical stimulation in culture process promotes extracellular matrix synthesis and leads to better function. Therefore, it is necessary to study the optimum conditions of mechanical stimulation.

Gaining better understanding of the mechanical and functional environment around chondrocytes is crucial to the clarification of the mechanism of osteoarthritis pathogenesis, the assessment of mechanical properties of the regenerated cartilage, and the clarification of the optimum condition of mechanical stimulation on the metabolism of chondrocytes.

The purpose of this study is to clarify the depth-dependent and time-dependent behaviors of local stress and strain in articular cartilage to understand the mechanical environment around chondrocytes in articular cartilage. For the numerical analysis based on biphasic finite element method to consider the coupled behavior of the solid phase and liquid phase (Mow et al. ${ }^{(1)}$ ), we investigated the depth-dependence and time-dependence of the elastic property in articular cartilage. As an approach, first, unconfined compression test of the cartilage tissue was performed and the local strain was estimated by measuring the changes in distance between corresponding chondrocytes stained with Calcein-AM (Murakami et al. ${ }^{(2)}$ ). Next, two-dimensional FEM based on biphasic theory was applied to simulate actual behaviors.

\subsection{Articular cartilage tissue}

Articular cartilage is composed of chondrocytes and an extracellular matrix. Extracellular matrix is mainly composed of proteoglycan and collagen, and produced by chondrocytes to provide scaffold to chondrocytes and support load to cartilage. Proteoglycan is highly hydrophilic and therefore can store a large amount of water and contribute to viscoelasticity. The hydrated proteoglycan is distended but confined by collagen network, and thus it supports the compressive load, while the collagen is given under pulling coercively.

The uppermost superficial layer of about 200 to $500 \mathrm{~nm}$ in cartilage tissue is called lamina splendens, and there are no chondrocyte and no collagen fibril within this layer. The uppermost superficial zone is covered with the adsorbed film consisting of phospholipids, protein, and glycoprotein complex. The proteoglycan-derived gel layer is located underneath adsorbed film in lamina splendens (Gardner et al. ${ }^{(3)}$, Higaki et al. ${ }^{(4)}$ ) and preserve low shearing resistance and protect the bulk body of cartilage (Murakami et al. ${ }^{(5)}$ ). A collagen layer resides at approximately 200 to $500 \mathrm{~nm}$ under this film and chondrocytes exist under this collagen layer.

Cartilage is categorized into the surface, middle and deep zones, and calcified zone by the gradient direction of collagen fibrosis, the morphology of the cells, and the presence or absence of calcareous deposits (Fig.1). Consequently, mechanical properties are different in the location. 
Alexopoulos et al. ${ }^{(6)}$ investigated an influence of Young's modulus distribution in extracellular matrix on pericellular matrix. They calculated Young's modulus about each position on depth direction from the unconfined compression test and derived the far field strain using inverse relationship between Young's modulus and strain. As a result, it became clear that the strain which occurred in cartilage tissue is decreased with the depth.

The upper 10 to $20 \%$ of cartilage tissue is called surface zone. Collagen fibers in surface is finer (30 to $32 \mathrm{~nm}$ ) and denser than lower layer. The fiber placement is parallel to the articular surface and the surface has high tensile property. The morphology of the cell is flat or fuseau and the orientation of the long axis of the cell is parallel to the articular surface.

The middle zone is the thickest of all layers and accounts for 50 to $70 \%$ of cartilage tissue. The collagen fibers in the middle zone have a diameter of 30 to $80 \mathrm{~nm}$ and form a network in a three-dimensional arrangement. The shape of the cell is round or fuseau. Chondrocytes in this zone are larger than those in surface and are arranged perpendicularly or radially to the articular surface.

The deep zone comprises the lowest 20 to $30 \%$ of the cartilage tissue. The chondrocytes are spherical and larger than those of the middle zone. The cells are assembled as columnar structure perpendicular to the articular surface. Collagen fibers also are arranged perpendicular to the articular surface.

The calcification layer is the layer in which caustic lime is deposited in the matrix. Between the deep zone and calcified zone, there is a thin wavelike band called tidemark. The glycosaminoglycan content is higher here and possibly contributes to cartilage reconfiguration.

As described above, the composition of cartilage is inhomogeneous and extremely complex. Therefore, it is necessary to consider not only whole tissue but also local behavior to explain the functional environment.

Fig.1 Cross-section diagram of cartilage tissue

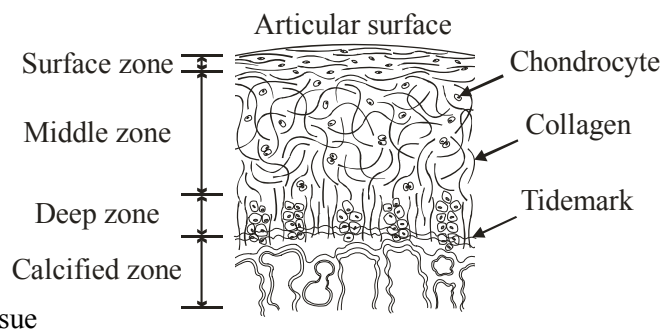

\subsection{The behavior of cartilage tissue to mechanical stimuli}

The structure and property of articular cartilage have depth-dependence. If the load acts on the tissue rapidly, the tissue does not have enough time to exude the interstitial fluid and thus presents similar behavior to the elastic body. While the load acts on the tissue slowly, the interstitial fluid is gradually exuded through the tissue. In compression, the permeability of cartilage becomes lower with an increase in compression strain (Jurvelin et al. ${ }^{(7)}$ ). When cartilage is compressed at high rate and kept at the definite position, the occurrence of peak stress and following stress relaxation behavior are observed as shown in Fig.2. In our unconfined compression test of $10 \sim 15 \%$ strain at $1000 \mu \mathrm{m} / \mathrm{s}$, peak stress ranged from 0.71 $\mathrm{MPa}$ to $3.76 \mathrm{MPa}$. To understand the mechanotransduction in chondrocytes in cartilage, the actual time-dependent and depth-dependent stress and strain around chondrocytes should be clarified.

The chondrocytes recognize their own deformation by the force to the cell membrane. We can estimate that the mechanical stimuli are transmitted by deforming chondrocytes with the deformation of extracellular matrix. It is believed that cytoskeleton, extracellular matrix receptor, and ionic change respond to the mechanical stimuli. In tissue engineering, it is noted that Young's modulus of regenerated cartilage increases by culturing under the 
periodic load condition or undergoing rehabilitation after transplantation. It is believed that the production is enhanced by mechanical stimuli to chondrocytes (Chowdhury et al. ${ }^{(8)}$ ).

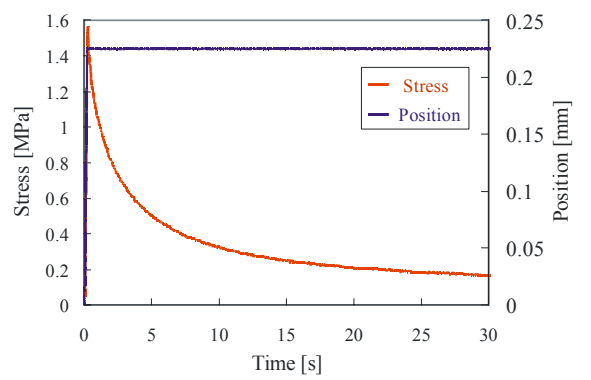

Fig. 2 Compressive behavior of articular cartilage to definite compressive deflection

\subsection{Composition of cartilage and biphasic theory}

Cartilage tissue is composed of chondrocytes and extracellular matrix with high water content and thus has biphasic properties. Biphasic theory is developed by Mow et $\mathrm{al}^{(1)}$. In this theory, cartilage tissue is modeled by acquiring the macroscopic characteristic of the tissue, and assumes that the soft tissue with high water content is composed of two types of phases, i.e., solid phase and fluid phase. Then, the fluid phase is able to flow at some level through the solid phase composed of porous material. Because this fluid flux makes resistance to compressive load in cartilage, the whole tissue presents viscoelastic behavior. This biphasic theory is appropriate to the expression of the stress relaxation behavior and the creep phenomenon. Thus, in this study, we conducted biphasic finite element analysis.

\section{Experimental methods for compression tests}

\subsection{Materials for compression tests}

Osteochondral explants were harvested from distal end of femur in porcine knee (6 to 7 months old). A $3 \mathrm{~mm}$ cylindrical core was punched from each osteochondral explant and then cut in half perpendicular to the articular surface with a scalpel blade(Fig.3). The samples were washed by PBS (Phosphate Buffered Saline;pH7.4, Invitrogen Corporation). Then, living cells as markers were stained with Calcein-AM $1 \mu \mathrm{L}$ (Molecular Probes) in $1000 \mu$ L PBS. The samples were incubated for $30 \mathrm{~min}$ at $37{ }^{\circ} \mathrm{C}$ and washed by PBS.

\subsection{Compression tester and experimental method}

In these unconfined compression tests on semi-cylindrical cartilage specimens, the changes in local strain were observed. The compression tester ${ }^{(2)}$ shown in Fig.4 is located on the stage of the confocal laser scanning microscope (CLSM, BIO-RAD, Radiance2000). The tester is constructed by impermeable alumina ceramic compressing plates, load cell, gap sensor for measuring the displacement of compressing plate, and linear actuator. Compression test is performed on the cover glass. The compressive behavior can be observed by object lens of CLSM. The position accuracy of $0.2 \mu \mathrm{m}$ and the maximum speed of $4000 \mu \mathrm{m} / \mathrm{s}$ are controlled by feed back system for the displacement of compressing plate correcting the changes in deformation of the load cell during stress relaxation.

First, a sample was set up on the cover glass, and soaked with PBS to keep it from drying out. The initial position was the position that the sample has contact with the impermeable compression plate and detective load of about $0.1 \mathrm{~N}$. The thickness of the tissue was estimated from cartilage surface to tidemark.

Under unconfined condition, the cartilage specimen was compressed to the defined total 
deformation at a constant velocity. After that, the position was kept during stress relaxation phase for $20 \mathrm{~min}$. Peak stress is maximum stress observed immediately after compression as shown in Fig.2. During compression tests, the compressive load was monitored and fluorescence images were continuously acquired. Changes in local strain were estimated from the changes in distances between corresponding cells.

In this study, we assume that semi-cylindrical specimen is nearly uni-axially compressed at equilibrium after stress relaxation in unconfined compression test and discuss mainly about the case of compression velocity of $1000 \mu \mathrm{m} / \mathrm{s}$ and the total compression rate of $10 \%$.

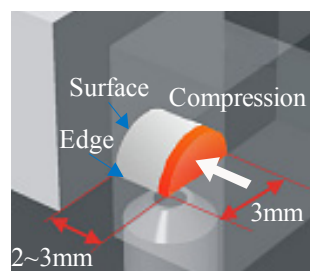

Fig. 3 Test specimen in compressive device.

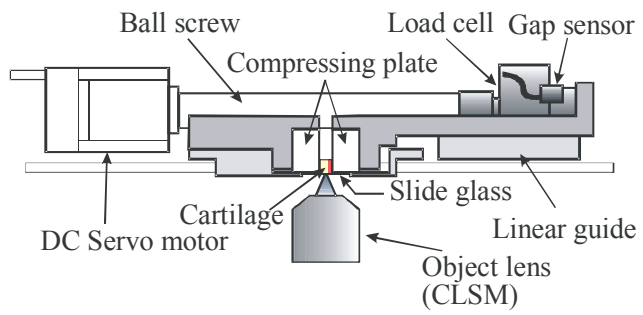

Fig.4 Compression tester located on microscope stage.

\section{Experimental results in compression tests}

\subsection{Changes in specimen profiles under compression}

The transmission images of the edge of the semi-cylindrical specimen before and after compression are shown in Fig.5. The lateral strain in surface zone is lager than those in deep zone. To estimate depth-dependent Young's modulus, we took notice of the fact that the pore pressure decreases little by little during stress relaxation process, and then the load is supported only by the solid phase in equilibrium. Thus, Young's modulus distribution was calculated based on the local strain in equilibrium.

(a)

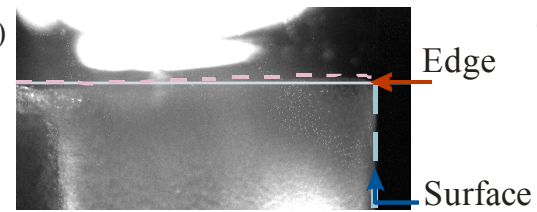

(b)

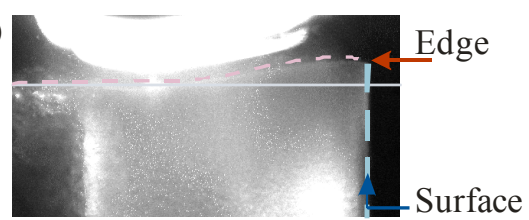

Fig. 5 Photograph of the edge of specimen. (a)Before compression. (b)Immediately after compression.

\subsection{Estimation of local strain depending on depth}

The local compression strain is calculated from the change in distance between the corresponding cells in the fluorescence images before and after compression. The fluorescence images before compression and at equilibrium observed by CLSM are shown in Fig.6. We could identify quite clearly the same cells, and measured the distance of the cell-to-cell. The cells in articular cartilage except surface zone moved mainly in the same direction as loading under unconfined compressive loading. At equilibrium without fluid pressure after stress relaxation, the lateral strain in surface zone was reduced to the lateral deformation to be defined by Poisson's ratio. The local compressive strain at equilibrium was calculated using Eq. (1), where $a$ and $b$ are the distance of the cell-to-cell before compression and one at equilibrium, respectively.

$$
\varepsilon=\frac{a-b}{a} \times 100
$$

We normalized the local compressive strain and plotted those against relative position $x$ in depth direction in Fig.7.The surface side is depicted as 0, and the deep zone end side is 1.

(a)

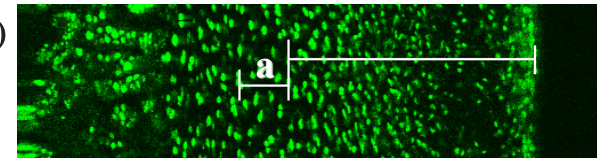

(b)

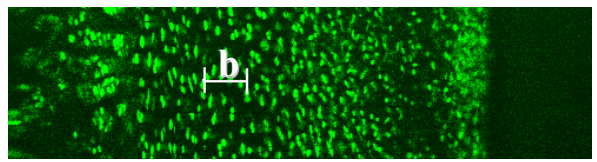

Fig. 6 Displacement of chondrocytes in cartilage. (a)Before compression. (b)At equilibrium. 


\subsection{Derivation of formula for depth-depending local strain distribution and Young's modulus distribution}

The approximate curve $\varepsilon(x)$ was fitted to the plot of local strain in Fig.7, where $\varepsilon_{0}$ is the average strain of the solid phase and $f(x)$ is the local strain distribution function. Immediately after compression, surface zone of sample swells in lateral direction due to fluid pressure. Then the intrinsic fluid flow gradually occurs, the fluid pressure relaxes and lateral strain is reduced. Although the final lateral strain becomes smaller, the lateral strain defined by Poisson's ratio of solid phase near surface increases according to an increase in compressive strain because Young's modulus of surface is lower than that of deep zone. After enough stress relaxation, fluid pressure becomes $0 \mathrm{MPa}$. At equilibrium, it is thought that the lateral bulge corresponds to the lateral deformation due to Poisson's ratio.

$$
\varepsilon(x)=\varepsilon_{0} f(x)
$$

We integrated Eq. (2) about overall range of cartilage tissue to calculate the average strain of the solid. Thus, the local strain distribution function is shown as Eq. (3).

$$
f(x)=\frac{\varepsilon(x)}{\varepsilon_{0}}
$$

We derived Young's modulus distribution function $\mathrm{g}(x)$ using inverse relationship between strain and Young's modulus for nearly uni-axial compression. At equilibrium, fluid pressure is 0 . Consequently, the local stress at $x$ of cartilage at equilibrium is equal to average stress. Thus, We obtain Eq. (4).

$$
g(x)=\frac{1}{f(x)}
$$

Therefore, Young's modulus depending on depth is defined by

$$
E(x)=E_{0} g(x)=\frac{E_{0}}{f(x)}
$$

In this study, we applied the compression velocity of $1000 \mu \mathrm{m} / \mathrm{s}$ and the compression strain of $10 \%$. The average strain of the solid phase $\varepsilon_{0}$ is $9.90 \%$ in this experiment. Mean value of the average Young's modulus $E_{0}$ of solid phase at equilibrium is $0.74 \mathrm{MPa}$. The local strain distribution function, and Young's modulus depending on the depth are Eq. (6) and (7), respectively.

$$
\begin{aligned}
& f(x)=\frac{46.2 e^{-6.53 x}+2.84}{9.90} \\
& E(x)=\frac{7.36}{46.2 e^{-6.53 x}+2.84}
\end{aligned}
$$

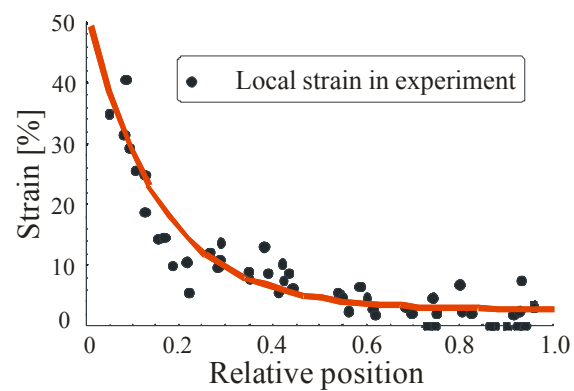

Fig.7 Local strain distribution.

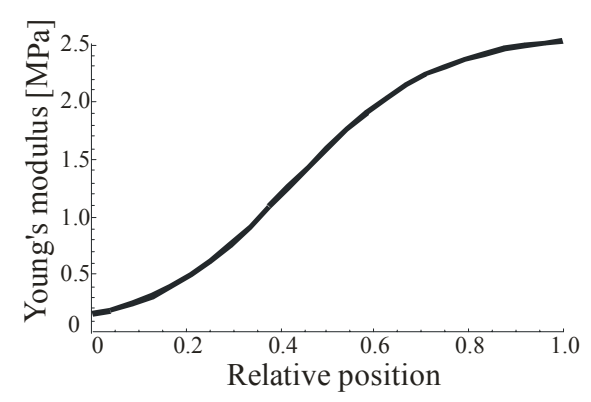

Fig. 8 Young's modulus distribution.

\section{Finite element analysis of articular cartilage model and boundary conditions}

\subsection{Finite element model}

General-purpose finite element analysis software (ABAQUS v.6.5) was used to analyze 
the biphasic model (Wu et. al. ${ }^{(9)}$ ).

The shape of articular cartilage model is rectangle that has $2 \mathrm{~mm}$ height and $3 \mathrm{~mm}$ width. We use two-dimensional model as the first step to future plan on multi-scale analyses to include chondrocyte. Characteristic behaviors in deformed profile, Mises stress and pore pressure obtained from two-dimensional model except small difference in stress and pressure values was verified by our related study using three-dimensional one. We modeled using biphasic fluid element (CPE8RP) that corresponds to quadrangle, 8-node, bilinear pore pressure and biquadratic displacement. The size of the each element corresponds to 10 $\mu \mathrm{m} \times 10 \mu \mathrm{m}$ and total number of element is 60,000 .

In this model, the upper $10 \%$ of cartilage tissue is surface zone, the lower $20 \%$ of the tissue is deep zone and middle zone is located between surface and deep zones. And the upper $10 \%$ of surface zone is defined superficial zone.

In this study, we created a homogeneous Young's modulus model (Model I ) and a depth-dependent Young's modulus model (Model II). Young's modulus of Model I was $0.74 \mathrm{MPa}$ as homogeneous value at equilibrium. In the case of Model II, Young's modulus is described as equation(7). Articular cartilage model was divided as 100 layers to depth direction and the corresponding Young's modulus was provided for each layer.

The void ratio $e$ is provided for ABAQUS by the following equation.

$$
e=\frac{d V_{v}}{d V_{g}+d V_{t}}
$$

Where $\mathrm{d} V_{v}$ is a volume of voids, $\mathrm{d} V_{g}$ is volume of grains of solid material, and $\mathrm{d} V_{t}$ is a volume of trapped wetting liquid.

In this study, we assumed that water content of $80 \%$ included in cartilage tissue is a volume of voids and other $20 \%$ is sum of the volume of grains of solid material and a volume of trapped wetting liquid. Thus, void ratio is 4 .

Poisson's ratio and permeability of solid phase were assumed as constant and used literature data shown in Table.1 (Guilak et al. ${ }^{(10)}$ ). The analysis was conducted for test duration of $1200 \mathrm{~s}$.

Table.1 Material properties.

\begin{tabular}{|c|c|c|}
\hline $\begin{array}{c}\text { Young's modulus } \\
\text { of solid phase, } E_{0}[\mathrm{MPa}]\end{array}$ & $\begin{array}{c}\text { Poisson's ratio } \\
\text { of solid phase, } v\end{array}$ & $\begin{array}{c}\text { Permeability } \\
k\left[\mathrm{~m}^{4} / \mathrm{N} \cdot \mathrm{s}\right]\end{array}$ \\
\hline 0.74 & 0.125 & $2.0 \times 10^{-15}$ \\
\hline
\end{tabular}

\subsection{Boundary conditions}

To compare experimental result with finite element analysis, the boundary condition for FEM was given to correspond to the compression test. FEM simulation of compression test was performed under compression velocity of $1000 \mu \mathrm{m} / \mathrm{s}$ and compression ratio of $10 \%$. Thus, amount of compression, compression velocity and compression time to definite deflection were $0.2 \mathrm{~mm}$ as $10 \%$ of thickness, $1000 \mu \mathrm{m} / \mathrm{s}$ and $0.2 \mathrm{~s}$, respectively.

The node of lower surface was confined and the displacements of the node in both $x$ and $y$ directions were 0 . The node of top surface was compressed at displacement of $0.2 \mathrm{~mm}$ and the transform in $y$ direction (parallel to surface) was unconfined. To simulate impermeable compressing plates in compression tester, water was assumed to seep only through right and left side of rectangle model.

\section{Result of FEM analysis}

\subsection{Comparison of experimental result with FEM analysis}

The transmission images of the specimen immediately after compression and its 
deformed profile immediately after compression of FEM simulation on homogeneous Young's modulus model and depth-dependent Young's modulus model are shown in Fig.9 (Murakami et al. ${ }^{(11)}$ ). Immediately after compression, the deformed profile of the depth-dependent Young's modulus model corresponded to actual profiles, while the homogeneous Young's modulus model showed barreled profile. From this result, the importance of the depth-dependence of Young's modulus was confirmed by compression test under unconfined condition.

Then, the total load capacity of Model II was compared with measured value by a load cell in experiment. The total load capacity estimated by FEM analysis is the sum of compression stress in vertical direction and pore pressure.

About all nodes, the stress at center of each element was averaged by area. In cases where average Young's modulus of solid phase is $0.74 \mathrm{MPa}$ obtained by compression test at equilibrium, the load carrying average stress by FEM analysis immediately after compression is about $0.17 \mathrm{MPa}$. On the other hand, experimental value of the average loading stress immediately after compression is about $2 \mathrm{MPa}$. Consequently, the total load capacity in FEM is about one order lower than the experimental one.

Therefore, we examined the effect provided by parameter such as permeability, void ratio and Young's modulus on the total load capacity immediately after compression. In this biphasic model, we changed the values in permeability from one-tenth to one thousandth and void ratio from 4 to 2.33, but these changes had little effect on stress level. In contrast, an increase as 1 order in Young's modulus had a large effect on stress enhancement.

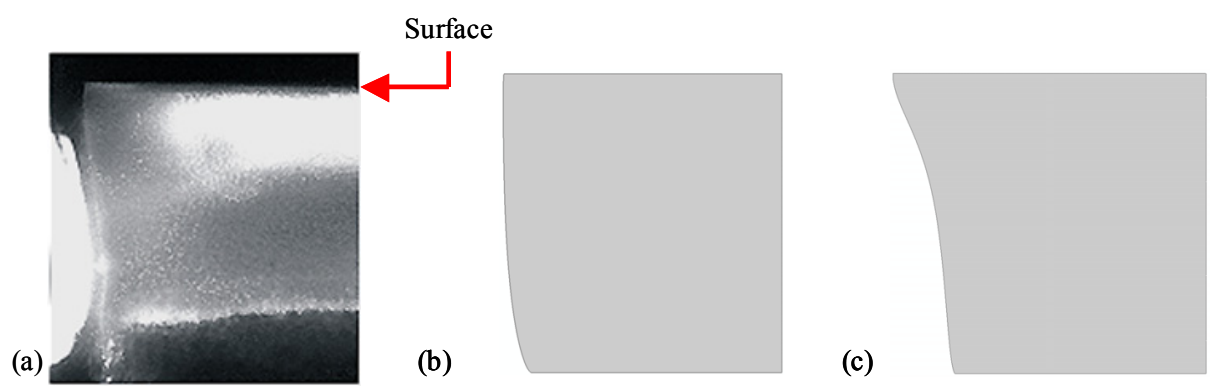

Fig.9 Profile of compressed cartilage. (a)Cartilage specimen.(b)Model I .(c)Model II .

\subsection{Effects of variation in Young's modulus on total load carrying capacity}

In Model II, it is thought that Young's modulus has a remarkable effect on stress enhancement immediately after compression. To conform the total load capacity immediately after compression to experimental value, it is necessary to increase as 12.74 times average Young's modulus at equilibrium $(0.74 \mathrm{MPa})$. Therefore, we created a model with average Young's modulus for solid phase of $9.47 \mathrm{MPa}$, and named Model III. The parameters except for Young's modulus are the same to those of Model II .

Compression stress, pore pressure for whole model and the total load capacity of Model II and Model III for half a minute after compression are shown in Fig.10. If average Young's modulus was increased, compression stress and pore pressure immediately after compression increased approximately 12.5 times and 10 times, respectively.

Thus, the loading average stress immediately after compression of Model III became 2 $\mathrm{MPa}$ and corresponded to experimental value. Deformed profiles and Mises stress distribution immediately after compression are shown in Fig.11. When average Young's modulus of solid phase was increased, not only stress of solid phase but also pressure of fluid one increased. Deformed profile of Model III immediately after compression was almost similar to that of Model II . Thus, the change in value of average Young's modulus of solid phase has little effect on deformed profile, because deformation profiles are controlled with relative distribution of elastic modulus under definite deflection. During stress 
relaxation, however, the total load capacity was maintained at higher level than experimental value. Therefore, we need to control the average Young's modulus.

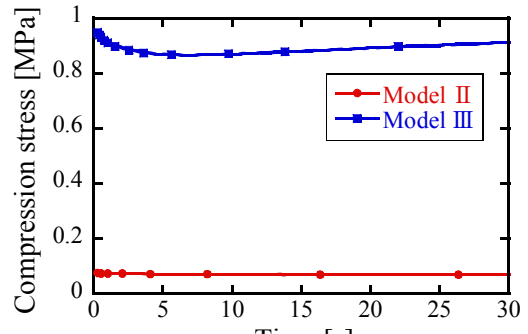

(a)

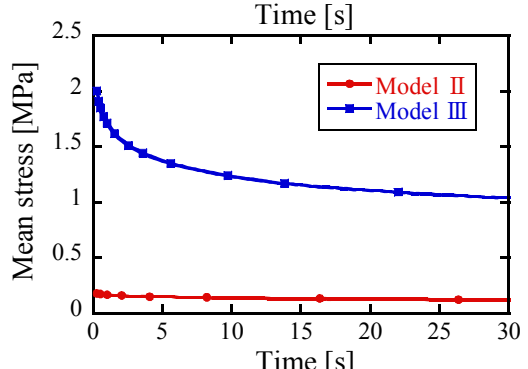

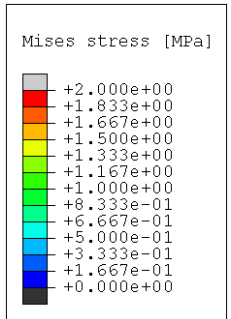

(a)

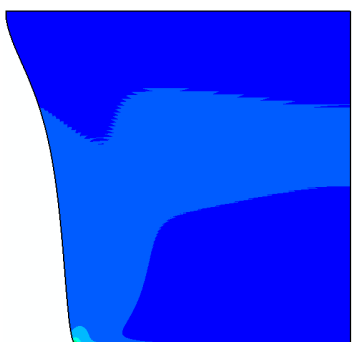

Fig. 1 $\left(E_{0}=0.74 \mathrm{MPa}\right)$. (b)Model III $\left(E_{0}=9.47 \mathrm{MPa}\right)$.

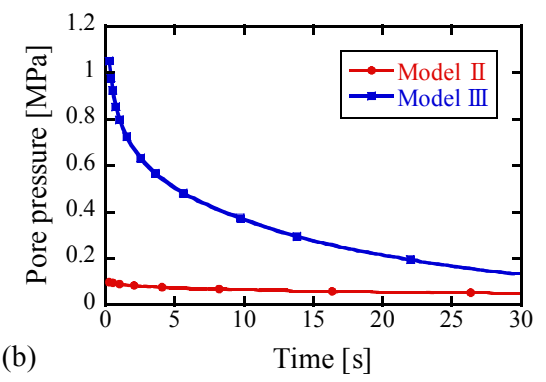

Fig.10 Comparison of stress and pressure. (a)Compressive stress.

(b)Pore pressure.

(c)Mean stress.

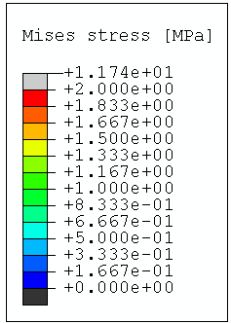

(b)

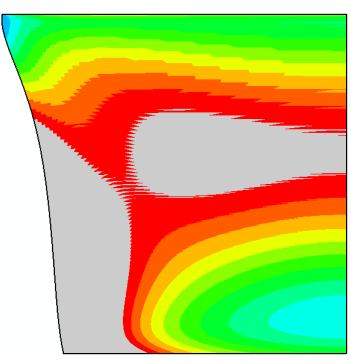

(a)Model II

\subsection{Cartilage tissue model considering the depth-dependence and time-dependence of elastic property}

To conform the total load capacity of FEM simulation based on biphasic theory to the experimental value, it is necessary to control elastic property in this FEM model.

Immediately after compression, because it has not enough time to flow intrinsic fluid in cartilage, whole tissue including intrinsic fluid shows the behavior like elastic body. Furthermore, the polymeric materials increase their stiffness at higher strain rate. Therefore, apparent Young's modulus is assumed to be larger than the equilibrium Young's modulus. During total deflection is maintained after compression, the intrinsic fluid flow gradually occurs, and the stress relaxes with decrease of apparent Young's modulus. After enough stress relaxation, an apparent Young's modulus becomes an equilibrium Young's modulus.

Consequently, we adopted instantaneous elastic modulus as an instantaneous ratio of stress to strain, created the model with gradual decrease in instantaneous elastic modulus, and named Model IV.

In unconfined compression test of articular cartilage at $1000 \mu \mathrm{m} / \mathrm{s}$, it was found that the total load capacity decreased close to that at equilibrium condition within $30 \mathrm{~s}$ after compression. Therefore, we changed elastic property from immediately after compression until about $30 \mathrm{~s}$ to conform FEM value to experimental value. When instantaneous elastic modulus of solid phase increases, we could increase the total load capacity without affecting deformed profiles. Therefore, we defined correction factor as function of time $T_{\mathrm{f}}$ and considered time dependence of instantaneous elastic modulus. The equilibrium elastic modulus, i.e., Young's modulus of $0.74 \mathrm{MPa}$ is denoted by $E_{\text {eq. }}$. Thus, instantaneous average elastic modulus can be written as equation (9). 


$$
E_{\text {inst }}=T_{f} \times E_{e q}
$$

Therefore, instantaneous elastic modulus is calculated by equation (10).

$$
E(x, t)=\frac{9.90 \times E_{\text {inst }}}{46.2 e^{-6.53 x}+2.84}
$$

First $T_{\mathrm{f}}$ was assumed and then $E_{\text {inst }}$ and $E(x, t)$ were calculated. FEM model was created using calculated $E(x, t)$ and the total load capacity was analyzed for a few seconds. During relaxation process, $T_{f}$ was modified if the difference of the total load capacity between FEM value and experimental one is more than $2 \%$. This method was repeated for approximately $30 \mathrm{~s}$ from immediately after compression.

The changes in instantaneous elastic modulus derived from FEM model in solid phase is shown in Fig.12. Between plotted values, the instantaneous elastic modulus was calculated by linear interpolation. The mean stress (total load capacity) and deformed profiles immediately after compression and Mises stress distribution of FEM simulation on Model II and IV are shown in Fig.13 and Fig.14, respectively. FEM value of the total load capacity corresponded to experimental one by providing time dependence to elastic property of solid phase. Though elastic property of solid phase changes, the deformed profile immediately after compression kept effect of depth-dependence and corresponded to experimental result.

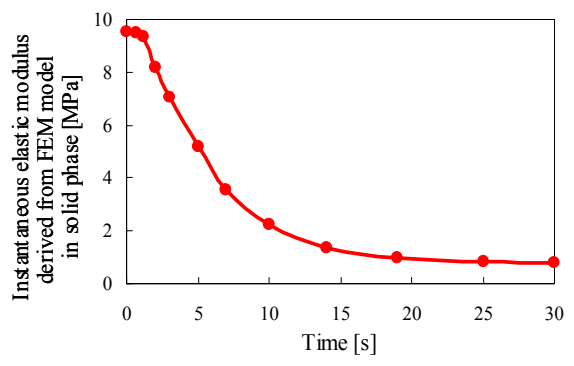

Fig.12 Time-dependent elastic modulus of modelIV.

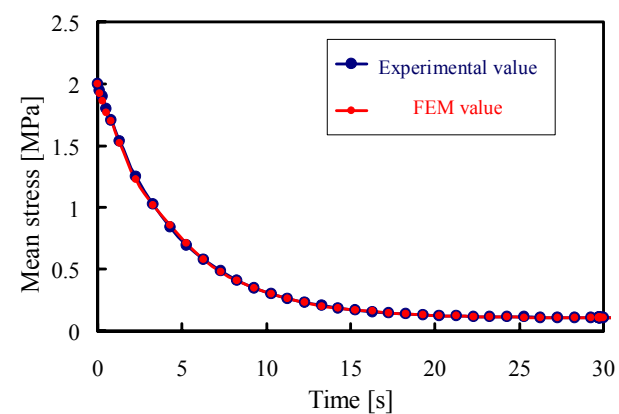

Fig. 13 Mean stress

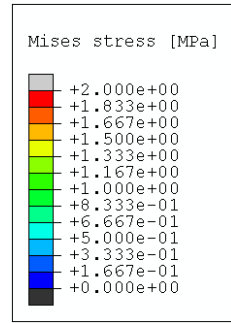

(a)

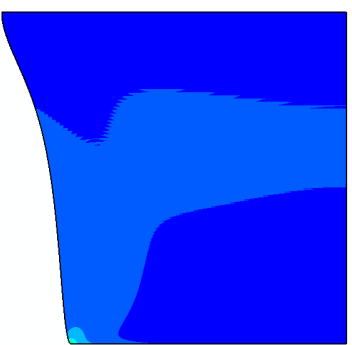

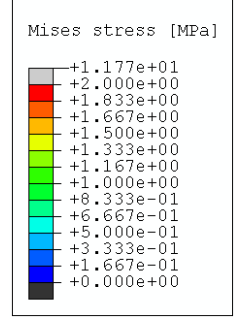

(b)

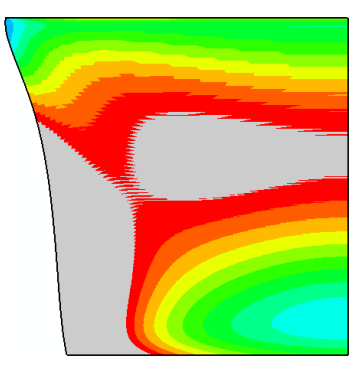

Fig.14 Profiles and Mises stresses of the models immediately after compression. (a)Model II $\left(E_{0}=0.74\right.$ MPa).(b)Model IV( $E_{0}$ :Time-dependent).

\section{Discussion and conclusions}

In this study, we performed unconfined compression test of articular cartilage at definite total deformation in compression tester located on the stage of CLSM and evaluated the changes in local strain by observing the changes in cell-to-cell distance in fluorescent images of stained chondrocytes. After stress relaxation, it was shown that Young's modulus of solid phase has depth-dependent distribution. For the compression velocity of $1000 \mu \mathrm{m} / \mathrm{s}$ and average compression of $10 \%$, most of the strain occurred in surface at equilibrium after stress relaxation. The superficial layer was strained to $50 \%$ after stress relaxation, while the deep zone was strained at about only $3 \%$ (Fig.7).

Next, to simulate the depth-dependent and time-dependent compression behaviors of articular cartilage in experiment, we applied a finite element analysis based on biphasic 
theory by using a model considering Young's modulus distribution depending on the depth. Then, we compared FEM result with experimental one. In this time, we focus on deformed profile immediately after compression, peak stress, and stress relaxation curve.

If solid phase of the model was provided as depth-dependent Young's modulus estimated from local strain distribution at equilibrium in compression test, the deformed profile of depth-dependent Young's modulus model immediately after compression corresponded to actual profiles. Consequently, we confirmed that Young's modulus has a distribution in the depth direction. In contrast, the total load capacity in FEM analysis was about one order lower than the experimental one. Then, we considered about the effectiveness of changes in permeability, void ratio, and Young's modulus to explain what is the most important factor about stress development.

First, we decreased permeability, but a decrease in permeability had no direct effect on stress development. Next, we decreased void ratio, but both the total load capacity and deformed profiles little changed.

In contrast, an increase of one order in Young's modulus had a remarkable effect on peak stress at full compression. To conform FEM result of the total load capacity to experimental one, we need to use 12.74 times increase of equilibrium Young's modulus of the solid phase. In this case, not only the stress of solid phase but also the pressure of fluid phase increased, and the load-carrying average stress of the model became $2 \mathrm{MPa}$ and corresponded to experimental value. Deformation profiles immediately after compression was almost similar to those in the previous model with depth-dependent equilibrium Young's modulus. Thus, it is thought that an increase in Young's modulus have no direct effect on deformed profile at full compression.

To correspond the load relaxation in FEM analysis to the behavior in experiment, time-dependent control of instantaneous elastic property was required. It is supposed that time-dependent decrease in instantaneous elastic modulus is partly derived from the gradual exudation of fluid from proteoglycan units surrounded collagen networks. However, as pointed out by Li et al. ${ }^{(12)}$, the nonlinear fibril reinforced poroelastic model is capable of describing the strong relaxation behavior and stiffening of cartilage in unconfined compression, although the compression rate at their condition is low as $1 \mu \mathrm{m} / \mathrm{s}$. Therefore, the tensile property of collagen networks is likely to play an important role in organization of stress-strain state in articular cartilage. We are evaluating the influence of addition of collagen spring element to biphasic element in FEM analysis and plan to report a detailed result in next paper.

In this study, we compared experimental results to FEM analysis and found the following facts.

(1) At high compression speed such as $1000 \mu \mathrm{m} / \mathrm{s}$, the high peak stress and the following stress relaxation was observed. The local strain showed depth-dependent and time-dependent behaviors. During stress relaxation, the superficial layer was largely strained, but the local strain in deep zone was recovered to lower level.

(2) If we provide depth-dependent Young's modulus distribution estimated from local strain at equilibrium to FEM analysis, the deformed profile of depth-dependent Young's modulus model corresponded to actual profiles. Consequently, we confirmed that Young's modulus has depth-dependent distribution. In contrast, the total load capacity estimated from FEM analysis is about one order lower than the experimental one.

(3) In this biphasic model, changes in permeability and void ratio had little effect on stress level. On the contrary, an increase in Young's modulus had a large effect on stress enhancement at definite compression. To conform FEM result of the total load capacity to experimental one, we needed to use 1 order higher value than equilibrium Young's modulus of the solid phase for the condition shown above. The deformed profile of edge part corresponded to experiment. 
(4) To conform the total load capacity of FEM simulation based on biphasic theory to load relaxation in compression test, it was necessary to decrease instantaneous elastic modulus as depending on the time.

At next stage, the role of spring elements of collagen should be discussed in compression test for consideration of actual anisotropy and inhomogeneity in cartilage.

\section{Acknowledgement}

Financial support was given by the Grant-in-Aid of the Ministry of Education, Culture, Sport, Science and Technology (Priority Area, No. 15086212).

\section{References}

(1) V. C. Mow, S. C. Kuei, W. M. Lai, C. G. Armstorng, Biphasic creep and stress relaxation of articular cartilage in compression theory and experiment, Journal of Biomechanical Engineering, Vol.102, pp.73-84, 1980

(2) T Murakami, N Sakai, Y Sawae, K Tanaka and M Ihara, Influence of Proteoglycan on Time-Dependent Mechanical Behaviors of Articular Cartilage under Constant Total Compressive Deformation, JSME International Journal, Series C, Vol.47, pp1049-1055, 2004

(3) D. L. Gardner, P. O'connor, J. F. S. Middleton, K. Oates, C. R. Orford, An investigation by transmission electron microscopy of freeze replicas of dog articular cartilage surface: the fibre-rich surface structure, Journal of Anatomy, Vol.137, No.3, pp.573-582, 1983

(4) H Higaki, T Murakami, Y Nakanishi, H Miura, T Mawatari, Y Iwamoto, The lubricating ability of biomembrane models with dipalmitoyl phosphatidylcholine and $\gamma$-globulin, Proceedings of the Institution of Mechanical Engineers, Part H: Journal of Engineering in Medicine, Vol.212, pp.337-346, 1998

(5) T Murakami, Y Sawae, M Ihara, The Protective Mechanism of Articular Cartilage to Severe Loading: Roles of Lubricants, Cartilage Surface Layer, Extracellular Matrix and Chondrocyte, JSME International Journal, Series C, Vol.46, No.2, pp.594-603, 2003

(6) L. G. Alexopoulos, L. A. Setton, F. Guilak, The biomechanical role of the chondrocyte pericellular matrix in articular cartilage, Acta Biomaterialia, Vol.1, pp.317-325, 2005

(7) J. S. Jurvelin, M. D. Buschmann, E. B. Hunziker, Mechanical anisotropy of the human knee articular cartilage in compression, Proceedings of the Institution of Mechanical Engineers, Part H: Journal of Engineering in Medicine, Vol. 217, pp215-219, 2003.

(8) T. T. Chowdhury, D. L. Bader, J. C. Shelton, D. A. Lee, Dynamic Mechanical Compression Applied in a Continuous or Intermittent Manner Influence Chondrocyte Metabolism, 2003 Summer Bioengineering Conference, pp.0185-0186, 2003

(9) J. Z. Wu, W. Herzog, M. Epstein, Evaluation of the finite element software ABAQUS for biomechanical modeling of biphasic tissues, Journal of Biomechanics, Vol.31, pp.165-169, 1998

(10) F. Guilak, Van C. Mow, The mechanical environment of the chondrocyte: a biphasic finite element model of cell-matrix interactions in articular cartilage, Journal of Biomechanics, Vol.33, pp.1663-1673, 2000

(11) T. Murakami, N. Sakai, Y. Sawae, M. Okamoto, I. Ishikawa, N. Hosoda, E. Suzuki, Depth-dependent compressive behaviors of articular cartilage and chondrocytes, BIOMECHANICS AT MICRO- AND NANOSCALE LEVELS, Vol.4, pp. 36-46, 2007

(12) L. P. Li, J. Soulhat, M.D. Buschmann, A. Shirazi-Adl, Nonlinear analysis of cartilage in unconfined ramp compression using a fibril reinforced poroelastic model, Clinical Biomechanics, Vol.14, pp. 673-682, 1999. 